\title{
Celebrate and support peer reviewers
}

\author{
As Peer Review Week approaches, Nature Geoscience takes the opportunity to thank its peer reviewers and \\ contemplate how their vital work can be better supported.
}

P eer reviewers are the backbone of the scientific literature, vetting papers for technical validity and guiding authors in strengthening their conclusions and interpretations. Each research paper and review article published in Nature Geoscience has been seen by at least two peer reviewers, usually more. And our published papers typically go through at least two rounds of review each (and that doesn't count all the rounds of review for the papers that do not make it into the pages of the journal). This represents an enormous effort by the geoscience community, and in honour of this year's Peer Review Week (10-15 September 2018), we look at how we provide - and can improve - support for these efforts.

One key challenge that we identified last year is a lack of diversity in the pool of current peer reviewers. At Nature Geoscience (Nat. Geosci. 10, 615; 2017), Nature and Nature Communications, the gender and geographic composition of our peer reviewers is far less diverse than that of our authors (https://go.nature. $\mathrm{com} / 2$ whhilZ). We were apparently not the only editorial teams to notice this disparity: the theme of Peer Review Week 2018 is 'Diversity in Peer Review'.

A lack of diversity in the pool of peer reviewers used to assess geoscience papers not only means that published papers are not benefiting from the full expertise of the scientific community, but also that large swaths of researchers are not receiving the benefits of being a peer reviewer. Peer reviewers report material benefits such as seeing work before it is published and improving their own writing skills. But, more importantly, they also see value in playing a role in the broader scientific community and helping to improve manuscripts (https://go.nature. com/2KSa5up; see 'Motivations and support supplement' supplement).

Since crunching the numbers last year, we have challenged our authors to make an effort to ensure that their reviewer suggestions reflect the gender and geographic diversity of the field. A spot check of recent submissions shows that some authors have risen to the challenge, at least in terms of gender. But suggestions for reviewers outside Europe and

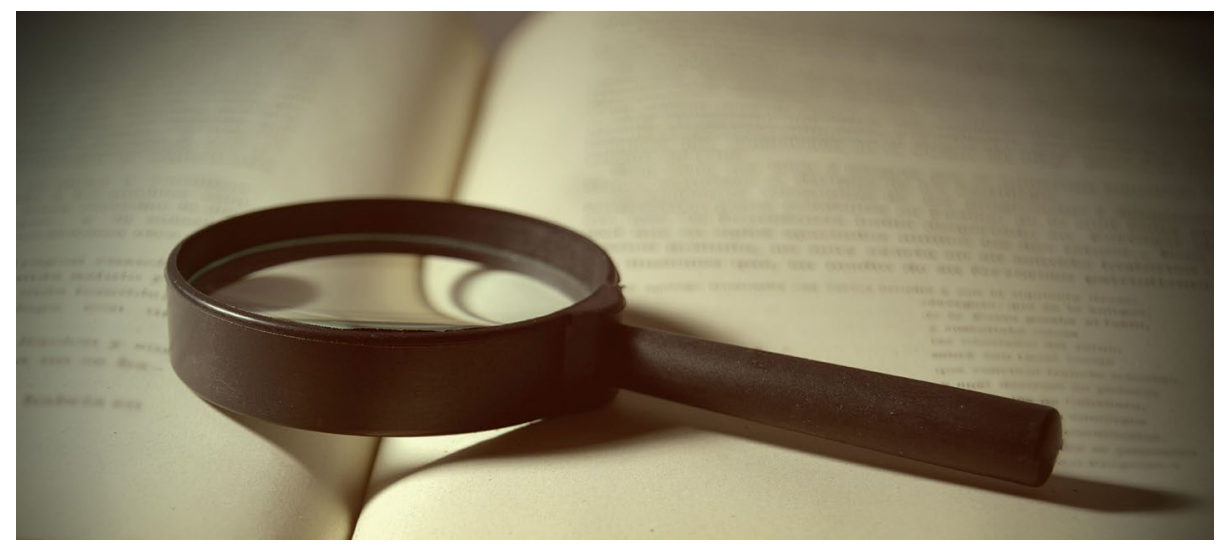

Credit: Juan Diego Oliva Plaza / Alamy Stock Photo

North America remain rare. Our editorial team is therefore working to expand our own networks of potential reviewers. Doing so will allow us to meet our primary aim of finding reviewers with the expertise required to assess the paper, making the best possible use of the experts scattered across the globe.

There are now resources available from Nature Research to assist the new reviewers we recruit, and those who might want a quick refresher: we have worked with colleagues to provide peer review training through the Nature Masterclass platform (https://go.nature.com/2nIjBXZ). This free series of videos and activities provides guidance on how to be a good peer reviewer, and also highlights some time-consuming pitfalls that befall nascent reviewers, such as extensively checking and correcting grammar and language.

Authors can also do their part to help smooth the peer review process. This support starts with writing the paper, focusing on clarity and justification for methods and assumptions and providing all the requisite supporting information at the initial submission. It also means ensuring that a paper really is publicationready - our editors on occasion receive complaints from our peer reviewers that a manuscript seems rushed, or is lacking vital experimental work that would be required to meet publication standards in any journal. Such premature submissions require reviewers to spend far more time than average, and may then require the attention of more researchers if the manuscript is then submitted to another journal.

Reviewers around the globe and across disciplines spend an average of four to six hours per manuscript (https://go.nature. $\mathrm{com} / 2 \mathrm{KSa} 5 \mathrm{up}$ ), and review across multiple titles. And we realize that many of our reviewers also lend their time and expertise as reviewers for other Nature titles too. Nature Geoscience and the whole of Nature Research are committed to recognizing reviewers for this work. Reviewers can download certificates that document the number of reviews performed for Nature journals from our online submission system (https://go.nature.com/2vLO9wg). We are also happy to provide verification for our peer reviewers who use Publons (https://go.nature.com/2Msnj5S) to record their peer review activities, and we have personally reached out to thank excellent and frequent reviewers for the Nature titles.

Every editor can point to a paper that was truly elevated through the efforts of its peer reviewers. They can also point to cases where hard work by reviewers prevented problematic studies from entering the scientific record. For this service to our journal and to the broader geoscience community, we thank you.

Published online: 31 August 2018 https://doi.org/10.1038/s41561-018-0228-z 